\title{
LES ROLES DES ACTEURS DANS LA FORMATION ET LE DEVELOPPEMENT DU CROWDLENDING : ANALYSE DES LIENS ET ECHANGES SOCIAUX
}

\section{Nathalie Duran, MCF Finance, IAE Réunion}

Résumé : Le crowdlending est une forme de financement participatif qui permet aux entreprises d'emprunter auprès de la foule par le biais d'une plateforme sur internet. Nous explorons les communautés de prêt et le rôle des acteurs par une recherche qualitative qui s'appuie sur des entretiens semi-directifs et une netnographie. Les résultats soulignent les opportunités du crowdlending sur le développement de l'économie et relèvent l'importance de la réciprocité dans cette communauté digitale de prêt qui dépasse l'aspect strictement financier.

Mots clés : crowdlending, communauté de prêt, échange social, parties prenantes, marché biface.

\begin{abstract}
Crowdlending is a finance process that allows individuals to lend money to businesses in a Peer to Business (P2B investor) approach on a web platform. We focus on crowdlending community. Our analysis relies on a methodology using an exploratory qualitative research joining interview and netnography as an innovative approach. The results highlight the potential of Crowdlending as complementary or even sometimes substitute of the traditional bank channel and the important role played by the community loan and the social exchange.
\end{abstract}

Key words: crowdlending, loan community, social exchange, stakeholders, two-sided market.

Je remercie les évaluateurs pour leurs commentaires et conseils avisés, ainsi que Djamchid Assadi, Rédacteur invité, pour ses précieuses suggestions. Je remercie également les personnes présentes au congrès de l'ISTEC le 8 décembre 2016 qui m'ont permis d'amélirorer la communucation presentée ce jour afin de publier mes travaux dans cette revue.

«Avec Lendopolis votre argent ne dort pas sur un Livret A ou sur votre assurance vie. Il travaille pour des PME françaises! Vous prêtez en direct aux entreprises de votre choix et recevez jusqu'à $12 \%$ d'intérêts chaque mois $"{ }^{1}$. La plateforme Lendopolis, créée par Vincent Ricordeau ${ }^{2}$, est spécialisée en projet d'entreprises et permet aux TPE et PME de plus de deux ans d'obtenir des prêts pouvant aller jusqu'à 2,5 millions d'euros ${ }^{3}$ par le biais d'Internet en dix jours en moyenne. Ce phénomène digital, appelé crowdlending, vient conférer un nouveau rôle aux Français : confier leur épargne à des entrepreneurs sous forme de micro-prêts ${ }^{4}$. Il peut s'agir de financer de l'immatériel, du BFR, des emplois, ou même encore du stock ou de la recherche et développement. Le financement participatif est en plein essor, le don (avec ou sans contrepartie) a augmenté de 36\%, tout comme l'investissement en capital, et le prêt (avec ou sans intérêts) connaît la plus forte progression avec $46 \%{ }^{5}$. En 2016, en crowdlending, 83 millions d'euros ont été collectés, ce qui représente 2.6 fois plus qu'en 2015 et les plateformes françaises ont battu un record en mai 2017, en finançant 70 projets à hauteur de 15 millions d'euros ${ }^{6}$. Ce financement est encore marginal puisque les banques

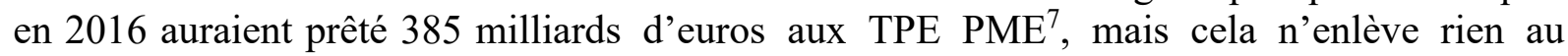
caractère exponentiel de sa

\footnotetext{
${ }^{1}$ Courriel envoyé par le groupe les Ecchos le 5 décembre 2015 à sa base de données de particuliers.

${ }^{2}$ V. Ricordeau est un acteur majeur de FP en France. C'est le co-fondateur de KissKissBankBank, plateforme française leader dans le don, et Hellomerci plateforme de prêts solidaires entre particuliers remboursant le capital sans intérêts. Avec ces 3 plateformes, le groupe est désormais le leader français du financement participatif.

${ }^{3}$ En moyenne le montant emprunté sur Lendopolis est de $72840 €$ au 17 juin 2017.

${ }^{4}$ Le prêt moyen sur Lendopolis s'élève à $165 €$ au 17 juin 2017.

${ }^{5}$ Etude Financement Participatif France et KPMG : crowdfunding 2016

${ }^{6} \mathrm{http}: / /$ www.crowdlending.fr/barometre-du-crowdlending /

${ }^{7}$ Communiqué du 22/02/2017 de la Fédération Bancaire Française
} 
croissance. Le crowdlending est d'ailleurs soutenu par le gouvernement. Le $1^{\text {er }}$ octobre 2014, un décret a permis la transformation digitale du prêt rémunéré et désormais un particulier peut prêter jusqu'à $2000 €$ par projet.

Cependant le coût du crédit est élevé et les premiers incidents de remboursements viennent entacher ce phénomène. Les prêteurs pointent du doigt la sincérité des informations financières fournies par les entreprises sur les plateformes, ils se sentent trahis par une communication qui enjoliverait la situation réelle et déplorent le manque de contrôle « post-financement ». En tant que tiers de confiance, et garant de l'information financière, l'expert-comptable semble avoir un rôle à jouer dans le crowdlending, comme en témoigne le partenariat récent du Conseil Supérieur de l'Ordre des Experts Comptables (CSOEC) avec certaines plateformes. Il pourrait constituer un lien de confiance entre les différentes parties prenantes (Duran, 2016).

Cette recherche vient dans le prolongement de nombreuses études exploratoires que nous menons pour mieux comprendre le crowdlending. Elle soulève la problématique suivante : quel rôle ont les acteurs de la communauté de prêt dans la croissance du crowdlending ?

La première partie présente le lien entre le crowdlending et la théorie de l'échange social. Puis nous détaillons la démarche qualitative exploratoire, pour finir par la présentation des résultats émergents, ainsi que des pistes de recherches futures.

\section{CROWDLENDING ET ECHANGE SOCIAL}

\subsection{Une nouvelle forme d'intermédiation}

Le crowdlending trouve ses origines dans le concept plus large du crowdfunding, qui lui-même s'intègre dans le crowdsourcing (Kleeman et al. 2008, Poetz et Schreier, 2012, Dardour 2015). Howe (2006) fut le premier à définir le crowdsourcing par «le fait de prendre un travail habituellement réalisé par un agent désigné - dans notre cas un banquier - et de l'externaliser à un groupe important de personnes sous la forme d'un appel ouvert à contribution ».

De nombreux auteurs ont apporté des définitions qui se rejoignent concernant le crowdfunding (Lambert et Schwienbacher 2010, Valanciene et Jegeleviciute 2013, Mollick 2014), en insistant sur la nécessité de prendre en considération les trois parties prenantes que sont: les investisseurs, les porteurs de projets et la plateforme intermédiaire. Pour Onnée et Renault (2013), le financement participatif « consiste pour un porteur de projet (...) à avoir recours aux services d'une plateforme de financement afin de proposer un projet auprès d'une communauté de contributeurs qualifiés de soutiens (backers) en échange éventuellement de contreparties préalablement définies ». La littérature décrit quatre modèles de financement : le don, la récompense, le prêt (avec ou sans intérêts) et l'intéressement (Frydrych et al., 2014 ; Onnée et Renault, 2014). Le modèle que nous étudions est basé sur le prêt avec versement d'intérêts, le crowdlending.

Ce phénomène digital englobe les efforts communs de divers acteurs pour financer différemment les entreprises. Les individus sont en nombre relativement important, et avancent des contributions relativement modestes par le biais d'internet et sans intermédiaires financiers classiques (définition adaptée de Mollick, 2014). Les Français sont de moins en moins satisfaits par les taux de rémunération décroissants des livrets d'épargne classiques ${ }^{8}$ ou des divers

\footnotetext{
${ }^{8}$ Le taux de rémunération du livret A est passé $2.25 \%$ fin 2013 à seulement $0.75 \%$ en 2015
} 
placements bancaires traditionnels ${ }^{9}$. L'année 2015 est d'ailleurs une année record de décollecte pour le livret A, la plus forte depuis vingt ans. Certains Français chercheraient à « donner un sens à leur épargne ${ }^{10}$ tout en obtenant un meilleur rendement de leurs économies. Cependant en 2016, 340 milliards d'euros reposaient encore sur les comptes courants ${ }^{11}$. Le crowdlending manque encore de notoriété dans certaines régions.

Le développement de ce marché semble également s'expliquer par une défaillance du financement bancaire traditionnel, notamment sur certains besoins des TPE/PME (Golic, 2014). Les théories des marchés bilatéraux et de l'intermédiation financière sont centrales pour expliquer le crowdlending (Rocher et Tirol 2006, Haas et al 2014). Ce marché du prêt rémunéré nécessite deux clientèles différentes mais interdépendantes : les particuliers prêteurs, membres des plateformes et les entreprises, ayant un besoin de financement. La plupart des plateformes fixent le taux d'intérêts en fonction du risque estimé et prélèvent des frais aux entrepreneurs. L'augmentation du nombre de projets présentés a tendance à développer le nombre de clients de l'autre côté du marché, c'est à dire à attirer la foule. Réciproquement, cette croissance du nombre de membres actifs peut aussi inciter les entreprises à choisir ce mode de financement. La plateforme est un intermédiaire qui permet de mettre les entreprises face à la foule, qu'elles doivent attirer et séduire. Elle ne se dégage un revenu que si elle parvient à attirer simultanément les deux clientèles, c'est à dire si la collecte aboutie. Cependant, les plateformes de crowdlending s'éloignent de la théorie de l'intermédiation classique du fait qu'elles ne détiennent pas les dépôts. Ce sont les prêteurs qui supportent les risques et qui gèrent leurs actes de prêt. (Duran et Dubrulle, 2017)

Bien que l'ensemble des études contribue à l'avancement des connaissances relatives à la compréhension du crowdlending, aucune à notre connaissance ne se réfère à la théorie de l'échange social, qui pourrait expliquer les relations au sein de la communauté de prêt et les rôles nouveaux de chacun.

\subsection{Lien avec la théorie des échanges sociaux}

La théorie des échanges sociaux (Mauss, 1950 ; Blau 1964 ; Cropanzano et Mitchell 2005) apparaît précieuse pour caractériser ces groupes. Il est indiscutable que le crowdlending repose sur un contrat économique mais nous cherchons à savoir si le groupe formant une communauté de prêt peut également être caractérisée par de l'interdépendance. L'échange social implique une réciprocité reposant sur trois principes fondamentaux : l'investissement, la confiance et l'engagement. Les membres de ce groupe doivent s'investir dans la relation afin d'en tirer les bénéfices. Il y a évidemment l'échange marchand qui consiste à confier son argent et attendre en retour sa restitution complétée d'intérêts. Cependant le comportement des particuliers peut ne pas être complètement rationnel. Leur investissement ne s'en tient pas au seul aspect financier, et ils peuvent rechercher une rétribution symbolique ou psychologique à leur action. Les prêteurs peuvent, par exemple, apporter des suggestions commerciales, ou encore des messages de soutien aux entrepreneurs. La plateforme fixe les critères de sélection et les analystes décident des projets qui seront mis en ligne, elle conditionne cet échange social. Elle peut être perçue comme un lieu de transactions sociales, qui engendrent des obligations de réciprocité. Les relations en son sein semblent aller au-delà du contrat économique à court

\footnotetext{
${ }^{9}$ Rendement moyen de l'assurance vie en $2015: 2.3 \%$. Le taux du PEL a quant à lui chuté de 2,5 à $1 \%$ en un an et demi.

${ }^{10}$ Vincent Ricordeau, entrevue vidéo du 5/02/2015

${ }^{11}$ Nicolas De Feraudy, à la 1 ère journée d'étude sur le crowdfunding le 9 juin 2017.
} 
terme, elles reposeraient sur des relations durables et des obligations mutuelles (Langevin et Mendoza, 2014).

Ces relations trouvent leurs origines dans la Rome antique, notamment dans l'analyse faite par Sénèque sur les Trois Grâces, qui illustrent selon lui les trois actions de la libéralité : « donner, recevoir, rendre ». Ces actions caractérisent parfaitement les échanges sociaux, selon Galoix et Lacroux (2010). Ils soulignent l'importance de l'investissement des partenaires, de leur volonté de s'engager durablement, afin de permettre la création de liens de confiance, nécessaires à la durabilité des liens à l'intérieur de la communauté.

L'investissement de la plateforme dans la relation avec la foule se définit par la volonté de protéger les divers intérêts. En effet les analystes sélectionnent rigoureusement les projets et ne publient que les moins risqués. Ils essaient de juger au mieux la capacité de l'entreprise à rembourser malgré le taux d'intérêts élevé et veillent à diminuer le risque de perte pour le particulier. Leur objectif est d'avoir le taux de défaut de paiement le plus faible ${ }^{12}$, afin de fidéliser les particuliers prêteurs. Le particulier a tendance à consulter les projets mis en ligne régulièrement et à prêter à nouveau dans une logique de réciprocité. La mise en place de critères rigoureux de sélection par les analystes de la plateforme dans le tri des projets et la présence de l'expert-comptable (EC) pour attester les informations financières fournies par les entreprises, pourraient ainsi inciter le prêteur à renouveler ses opérations de prêts.

Blau (1964) a également signalé le caractère central de la confiance. Premièrement, la présence de l'EC dans la relation et son implication dans le crowdlending devrait renforcer l'engouement pour le financement participatif. Il apporte de la crédibilité aux données financières communiquées, et permet d'améliorer la confiance accordée par le particulier concernant la communication des données financières. Dans leurs recherches Couteret et Audet $(2006)^{13}$ montrent qu'un expert-comptable impliqué adaptant et personnalisant son accompagnement à l'entreprise, impacte les pratiques du dirigeant de PME. Les dirigeants ont confiance en lui et sont influencés par ses conseils. Le partenariat avec l'ordre des EC renforce la réputation de la plateforme. Selon Cusin (2006), « la réputation est un élément de la confiance qui présuppose le rôle d'un tiers qui fournit des informations et/ou recommande le partenaire de l'échange » L'EC pourrait être un vecteur de croissance de ce mode de financement. De la même manière, l'expérience et les compétences des créateurs de la plateforme, peuvent générer la confiance des divers acteurs. La construction d'une relation de confiance mutuelle, entre les parties prenantes, peut inciter le particulier à réitérer ses opérations de prêts.

Depuis le lancement de Lendopolis en novembre 2014, la plateforme a permis de financer $11071700 €$ et permis à 152 projets d'entreprises en France de se réaliser ${ }^{14}$. Sa particularité est de placer l'EC au cœur du processus. Il doit attester les informations financières prévisionnelles de l'entreprise en amont. Lendopolis, tente de gagner en réputation par la confiance des investisseurs, d'une part avec l'expérience de ses fondateurs, mais également grâce à son partenariat avec la marque expert-comptable.

Enfin, Soulard (2015) s'appuie sur l'exemple du nombre de clients installés dans un restaurant qui le rend bien plus attractif, et affirme que certains internautes sont influencés favorablement par le nombre d'avis, "en dépit du risque avéré de manipulation ». De la même manière, le

\footnotetext{
${ }^{12}$ Le taux de défaut de paiement varie d'une plateforme à l'autre, $10,51 \%$ sur Lendopolis,

${ }^{13}$ Cités par Chapellier et al. (2007).

${ }^{14}$ Données recueillies sur la plateforme Lendopolis le 17 juin 2017.
} 
prêteur pourrait être influencé positivement par le nombre de prêteurs ${ }^{15}$, et par les divers échanges sur la plateforme. Il met en exergue également la crédibilité de la source à travers son expertise et sa fiabilité. Concernant la communauté de prêt, l'entreprise devra donc démontrer sa compétence, c'est-à-dire sa capacité à honorer ses engagements. La fiabilité sera appuyée par la transmission d'informations sincères et transparentes ${ }^{16}$. Le particulier, au-delà de l'aspect purement financier, pourra être influencé par la réputation de l'entreprise.

\subsection{Une relation tripartite particulière}

L'originalité du crowdlending repose sur l'intégration de la foule dans la prise de décision d'octroi de crédit à l'entreprise. Un partenaire nouveau vient compléter les parties prenantes traditionnelles : le particulier prêteur. La plateforme constitue également un acteur nouveau (Bessière et Stéphany, 2014), elle inclut le fondateur, les analystes financiers et les gestionnaires. La communauté de prêt s'alimente des effets de synergies entre : la plateforme (et ses dirigeants), les entrepreneurs (accompagnés de leur expert-comptable) et les microinvestisseurs (la foule).

Plusieurs études en marketing ont examiné les communautés de consommation et le phénomène de co-production de valeur (Cova 2008, Leroy 2008). Nous sommes en présence d'un processus similaire avec une «communauté de prêt » émergente. Le rôle de chacun dans la relation d'échange doit être clairement défini (Leroy, 2008). La communauté est composée d'une multitude d'individus hétérogènes, aux intérêts potentiellement divergents (Darras-Barquissau, 2011). Le particulier n'est plus seulement un consommateur, un producteur, ou un actionnaire, avec le crowdlending il endosse le rôle d'un financeur. Il devient un partenaire financier d'un nouveau type, qu'il faut comprendre pour mieux fidéliser.

Sur un processus similaire à celui des sociétés cotées qui s'adressent à leurs actionnaires, les TPE/PME, au-delà de la simple information financière, se doivent d'effectuer d'une part une véritable communication financière en incluant la dimension stratégique (Chekkar 2005), et d'autre part des éléments de nature extra-financière et qualitative (Cauvin et al. 2006). L'objectif est de mieux prendre en compte les attentes et les besoins de la communauté.

Le crowdfunding n'est pas seulement un financement mais aussi une information (Belleflamme et al., 2014). Il n'est pas restreint à un financement de proximité territoriale. « Il permet à des projets locaux d'attirer des investisseurs mondiaux » (Golic, 2014). Il favorise également la promotion de l'entreprise (Golic, 2014). Les projets exposés bénéficient d'une vitrine virtuelle consultée d'une part par la communauté mais aussi par des internautes curieux. Les prêteurs sont un second vecteur de propagande. Impliqués et attachés au projet financé, ils communiquent sur celui-ci via leurs propres réseaux sociaux (Gajda et Walton, 2013). Onnée et Renault (2013) affirment que le financement participatif apparaît comme un moyen de médiatisation, de communication qui permet à l'entreprise de se faire connaître et ainsi d'attirer de nouveaux particuliers. En outre, selon Kuti et Madarasz (2014), le financement participatif fonctionne comme les réseaux sociaux et permet au porteur de projet d'entrer en contact avec un large public intéressé par le projet, de communiquer et de créer des interdépendances. Cela confirme l'apport de la théorie de l'échange social. L'entrepreneur peut ainsi recevoir des encouragements ou des retours de la part des investisseurs. Il aura de ce fait, une meilleure connaissance des attentes des clients et pourra mieux cerner le profil des consommateurs. « $\mathrm{Si}$ de nombreuses personnes croient en une idée ou un projet, et que celui-ci est financé, c'est

\footnotetext{
${ }^{15}$ Toutes les plateformes affichent volontairement le $\%$ de fonds restants à collecter en temps réel pour influencer le prêteur.

${ }^{16}$ Ceci sera facilité par la présence de l'EC, dont c'est le métier.
} 
l'expression de la sagesse de la foule, ce projet ne peut alors que réussir » (Valanciene et Jegeleviciute, 2013). La campagne permet ainsi au dirigeant de la TPE/PME de tester la faisabilité de son projet et d'avoir un premier retour du marché. Les particuliers prêteurs n'ont pas toujours des comportements rationnels et peuvent se laisser influencer par leurs émotions, leurs affinités ou leurs goûts personnels.

Le fonctionnement des plateformes, telle que Lendopolis, emprunte des caractéristiques à la finance solidaire. Selon Artis (2009), la finance solidaire « regroupe un ensemble d'opérateurs non bancaires proposant des services financiers (prêt, épargne, garantie) à des porteurs de projets d'activités économiques suivant une logique de financement combinant des principes marchands et non marchands. L'enjeu est l'accès au crédit ou à la monnaie dans le cadre d'activités productives et l'adjectif solidaire qualifie l'entraide mutuelle entre les hommes, parties prenantes de la relation de financement ». Ici l' «entraide mutuelle » rassemble la TPE/PME, 1'EC, la plateforme et la foule au sein d'une communauté virtuelle, dont l'intelligence collective permet le financement de projets divers et variés.

\section{METHODOLOGIE QUALITATIVE DE LA RECHERCHE}

La démarche de recherche est qualitative et exploratoire. Elle repose sur des entretiens semidirectifs avec guide menés auprès des membres de la communauté de prêts, experts comptables, entrepreneurs ainsi que sur la netnograhie. La netnographie est une branche de l'ethnographie qui analyse le comportement libre des individus sur le net (Kozinets, 2002). Il s'agit d'une méthode qualitative employée principalement dans les recherches en marketing, basée sur les communautés virtuelles de consommation (Bernard, 2004). Cette méthode a été récemment appliquée pour une analyse du crowdsourcing sélectif (Renault 2014). Nous l'utilisons pour l'étude des communautés de prêt.

La recherche s'est déroulée en quatre étapes :

(1) En décembre 2014, le Conseil Supérieur de l'Ordre des Experts Comptables (CROEC) de La Réunion faisait part de son partenariat avec la plateforme Lendopolis. Des entretiens avec les membres du CROEC ont suggéré à la fois l'intérêt du crowdlending pour les entreprises et l'absence d'information et de connaissances concernant ces modes alternatifs de financement. Une recherche de nature exploratoire a donc débuté en mars 2015 par des entretiens auprès de la profession dans le département. L'objectif était de comprendre les perceptions des forces du crowdlending.

(2) Par la suite, trois entreprises ayant financé leurs projets via des plateformes de crowdlending ont été identifiées localement. Des entretiens ont été menés avec guide semi-directif auprès des dirigeants de janvier à mars 2016, afin de comprendre les raisons pour lesquelles ils ont choisi ce mode alternatif de financement.

(3) En raison de la petitesse de l'échantillon et du caractère récent du phénomène, la collecte des données primaires a été enrichie par une netnographie. Des recherches débutées fin 2014 se sont poursuivies jusqu'en mai 2016. Elles ont permis d'utiliser :

(a) des entretiens-vidéos sur différents sites internet comprenant des entrevues individuelles et collectives, ou des tables rondes lors de salon ou d'émissions télévisées ;

(b) des entretiens retranscrits d'entrepreneurs ; 
(c) des blogs sur le crowdlending, tel crowdlending.fr, créé par Mathieu George - un entrepreneur de 38 ans, fondateur du réseau Creditprofessionnel.com-, qui comprend un ebook (de septembre 2015), où sont compilés des entretiens menés auprès de prêteurs ou fondateurs de plateformes et d'autres informations sur le crowdlending en France ;

(d) des forums tels « Parlons de crowdlending en général » qui contenait en mai 2016, 49 sujets et 447 réponses portant majoritairement sur la sélection des entreprises, leurs défaillances, et des conseils pour la lecture des informations financières.

(4) Reposant sur les méthodes ethnographiques, la netnographie nécessite de s'immerger dans la communauté et de s'ancrer dans l'environnement virtuel. Par conséquent, nous nous sommes inscrits en tant que membre sur plusieurs plateformes de crowdlending françaises afin d'accéder aux informations disponibles et aux conversations virtuelles sur les forums. Plusieurs échanges ont eu lieu avec des analystes des plateformes, notamment la plateforme Lendopolis via le chat.

Tout au long des 18 mois, un journal de bord a été rédigé. Ce journal est chronologique, il comprend toutes les notes de terrain qui ont aidé à la réflexion, telles par exemple les évènements qui peuvent se produire (ex : premier défaut de paiement sur Unilend, premier retard sur Lendopolis), ou encore les conséquences de ces événements (ex : les réactions des prêteurs sur les blogs).

Le tableau 1 résume les différentes sources de données utilisées dans la recherche, analysées dans une perspective de compréhension, en vue de déterminer l'importance de la réciprocité dans les échanges sur les communautés de prêt.

L'analyse des données a suivi trois étapes : (1) L'ensemble des données brutes a fait l'objet de plusieurs lectures. (2) Les thèmes saillants ont été mis en évidence et ont conduit au développement d'une grille d'analyse heuristique, complétée après chaque analyse de documents -dont les entretiens- ; (3) les liens entre chaque thème ont été identifiés en vue de comprendre comment la communauté de prêt s'enrichit des échanges sociaux.

Tableau 1 : Sources des données.

\begin{tabular}{l|l|l|l|l}
\hline \multicolumn{1}{c|}{$\begin{array}{c}\text { Nature du } \\
\text { document }\end{array}$} & \multicolumn{1}{|c|}{ Date } & $\begin{array}{l}\text { Taille - } \\
\text { durée }\end{array}$ & \multicolumn{1}{c}{ Entrevue } & \multicolumn{1}{c}{ Commentaires } \\
\hline Entrevue vidéo & $26 / 11 / 2014$ & $8 \mathrm{~min}$ & $\begin{array}{l}\text { Vincent Ricordeau et } \\
\text { Nicolas Julhès }\end{array}$ & $\begin{array}{l}\text { BFM Business - porteur de projet } \\
\text { Algebra Drinks ayant levé 50 000€ }\end{array}$ \\
\hline Entrevue vidéo & $26 / 01 / 2016$ & $13 \mathrm{~min}$ & Nicolas Lesur & Fondateur Unilend - BFM Business \\
\hline $\begin{array}{l}\text { Table ronde } \\
\text { vidéo }\end{array}$ & $28 / 01 / 2016$ & $27 \mathrm{~min}$ & $\begin{array}{l}6 \text { intervenants dont } \\
\text { M. George, N. Lesur, } \\
\text { V. Ricordeau. }\end{array}$ & $\begin{array}{l}\text { Paris Fintech Forum : échanges sur } \\
\text { l'évolution du marché, les défauts de } \\
\text { paiements, etc. }\end{array}$ \\
\hline Webinar & $9 / 02 / 2016$ & 65 min & Vincent Ricordeau & Crowdlending.re par M. George. \\
\hline Webinar & $8 / 03 / 2016$ & 78 min & N. Lesur & Crowdlending.re par M. George \\
\hline Entrevue texte & $13 / 08 / 2015$ & 3 pages & JM Clauzel & $\begin{array}{l}\text { Métronome Technologies, 50000€ en 10 } \\
\text { jours sur Unilend }\end{array}$ \\
\hline Entrevue texte & $9 / 09 / 2015$ & 3 pages & Julie Machillot & $\begin{array}{l}\text { Fondatrice d'Emerite RH financement } \\
\text { complémentaire. Collecte en 24h au } \\
\text { taux de 7.7\% sur Unilend }\end{array}$ \\
\hline Entrevue texte & $12 / 10 / 2015$ & 3 pages & Eric Wagner & $\begin{array}{l}\text { Dirigeant d'Outils Précis ; 250000€ en } \\
28 \text { jours sur Unilend }\end{array}$ \\
\hline Entrevue texte & $4 / 11 / 2015$ & 3 pages & Gérard Dufour & $\begin{array}{l}\text { Dirigeant Société Prestiactes, nov 2014 } \\
\text { et juillet 2015 sur Unilend }\end{array}$ \\
\hline Entrevue texte & $25 / 11 / 2011$ & 3 pages & Pascal Tripon & $\begin{array}{l}\text { Société Pascal et Beatrix }-288000 € \text { en } \\
\text { deux fois, 50\% en autofinancement, taux } \\
9 \% \text { sur Unilend }\end{array}$ \\
\hline Entrevue texte & $5 / 01 / 2016$ & 3 pages & Thierry Compagnon & Illico Café 100 000€ sur Unilend \\
\hline
\end{tabular}




\begin{tabular}{|c|c|c|c|c|}
\hline Entrevue texte & $2 / 02 / 2016$ & 3 pages & François Chabanian & $\begin{array}{l}\text { Bel air fine Art, } 400000 € \text { sur } 1700000 € \\
\text { nécessaires ( } 2 \text { banques pour } 400000 € \\
\text { chacune) au taux de } 9.1 \% \text { sur Unilend }\end{array}$ \\
\hline Entrevue texte & $11 / 09 / 2015$ & 6 pages & Gilles Muller & $\begin{array}{l}\text { Edélices, Premier Projet financé par } \\
\text { Bolden, } 50000 € \text { à } 7.24 \% \text {, couplé à fonds } \\
\text { propres et crédit bancaire }\end{array}$ \\
\hline Entrevue texte & $29 / 09 / 2015$ & 4 pages & Colette Caron & $\begin{array}{l}\text { La table d'Antan, } 20000 € \text { sur } 48 \text { mois à } \\
8.94 \% \text { sur Bolden. }\end{array}$ \\
\hline Entrevue texte & $5 / 10 / 2015$ & 5 pages & Patrice Gauthier & $\begin{array}{l}\text { Savoir-faire de France, } 30000 € \text { sur } 36 \\
\text { mois à } 7.34 \% \text { sur Bolden }\end{array}$ \\
\hline Entrevue texte & $19 / 10 / 2015$ & 4 pages & Olivier Grosjean & $\begin{array}{l}\text { OG conseils services, prêt de } 15000 € \text { sur } \\
\text { un an à } 8 \% \text { sur Bolden }\end{array}$ \\
\hline Entrevue texte & $24 / 11 / 2015$ & 3 pages & JP Ouvrard & $\begin{array}{l}\text { Fondateur Axo et actifs, } 30000 € \text { sur } 36 \\
\text { mois à } 5.8 \% \text { sur Bolden }\end{array}$ \\
\hline Entrevue texte & $4 / 01 / 2016$ & 5 pages & Stéphane Charlier & $\begin{array}{l}\text { Willy Schneider } 20000 € \text { sur } 24 \text { mois à } \\
8.8 \% \text { sur Bolden }\end{array}$ \\
\hline Entrevue texte & $5 / 01 / 2016$ & 5 pages & Aurélien Bauters & $\begin{array}{l}\text { Co-fondateur les clés du monde, } 30000 € \\
\text { sur } 12 \text { mois à } 5.8 \% \text { sur Bolden }\end{array}$ \\
\hline Entrevue Texte & $19 / 01 / 2016$ & 6 pages & David Juhel & $\begin{array}{l}\text { Co-fondateur de The architects, } 15000 € \\
\text { à } 8 \% \text { sur } 12 \text { mois sur Bolden }\end{array}$ \\
\hline Article Unilend & $12 / 10 / 2015$ & 2 pages & Unilend.fr & $\begin{array}{l}\text { Promob honore ses commandes grâce au } \\
\text { CF }\end{array}$ \\
\hline Statistiques & $15 / 05 / 2016$ & 1 page & Unilend. & Période du 12/11/2013 au 30/04/2016 \\
\hline Statistiques & $15 / 05 / 2016$ & 1 page & Lendpolis & Période du 19/11/2014 au 14/05/2016 \\
\hline Article & $8 / 03 / 2015$ & 9 pages & Tristan Grué & $\begin{array}{l}10 \text { professionnels donnent leur avis sur } \\
\text { le crowdlending. }\end{array}$ \\
\hline Article & $3 / 12 / 2015$ & 5 pages & Tristan Grué & $\begin{array}{l}\text { Financer son entreprise : Bpi France ou } \\
\text { crowdlending? }\end{array}$ \\
\hline Echanges Blog & $22 / 02 / 2016$ & 4 pages & Crowdlending.fr & $\begin{array}{l}\text { Sélection des entreprises méthodologie - } \\
3 \text { réponses, } 4 \text { participants }\end{array}$ \\
\hline Echanges Blog & $\begin{array}{l}1 / 07 / 2015- \\
11 / 01 / 2016\end{array}$ & 7 pages & Crowdlending.fr & $\begin{array}{l}\text { Lancement } \mathrm{du} \text { baromètre } \mathrm{CL} \\
\text { entreprises. } 18 \text { réponses, } 7 \text { participants. }\end{array}$ \\
\hline Echanges Blog & $27 / 10 / 2015$ & 2 pages & Crowdlending.fr & $\begin{array}{l}\text { Commentaires sur « Pour être Bien chez } \\
\text { Soi ». } 6 \text { réponses, } 4 \text { participants }\end{array}$ \\
\hline Echanges Blog & $\begin{array}{l}22 / 10 / 2015 \\
30 / 11 / 2015\end{array}$ & 3 pages & Crowdlending.fr & $\begin{array}{l}\text { Pharmacie CWB :interactions prêteurs } \\
\text { pharmacien. } 4 \text { réponses, } 3 \text { intervenants }\end{array}$ \\
\hline Echanges Blog & $\begin{array}{l}13 / 07 / 2015 \\
25 / 02 / 2016 \\
\end{array}$ & $\begin{array}{l}27 \\
\text { pages }\end{array}$ & Crowdlending.fr & Groupama : 75 réponses 19 participants \\
\hline Echanges Blog & $\begin{array}{l}26 / 02 / 2016 \\
29 / 02 / 2016 \\
\end{array}$ & 6 pages & Crowdlending.fr & Rôle de l'EC : 9 réponses 4 participants \\
\hline Ebook & Sept 2015 & $62 \mathrm{p}$ & Mathieu George & Investir en CL : mode d'emploi. \\
\hline $\begin{array}{l}\text { Entretien semi- } \\
\text { directif }\end{array}$ & Mars 2015 & $30 \mathrm{~min}$ & Abdoullah Mollan & Trésorerier du CROEC \\
\hline Entretien SD & Mai 2015 & $110 \mathrm{~min}$ & Marcelino Burel & Président du CROEC de la Réunion \\
\hline Entretien SD & Mai 2015 & $45 \min$ & JL Chane Sam & Vice-Président du CROEC \\
\hline Entretien SD & Mai 2015 & $55 \min$ & Rémy Amato & Second Vice-Président du CROEC \\
\hline Entretien SD & Juin 2015 & $45 \min$ & Julie Lamarque & Présidente de l'ANECS \\
\hline Entretien SD & Juin 2015 & $60 \mathrm{mn}$ & Frederic Rossillon & EC nouvellement installé \\
\hline Entretien SD & Janv 2016 & $35 \min$ & Vincent Aubras & Dirigeant de Promob. \\
\hline Entretien SD & Janv 2016 & $45 \mathrm{~min}$ & M. ChoWingBom & Pharmacie CWB Belcour \\
\hline Entretien SD & Janv 2016 & $100 \mathrm{~min}$ & Isabelle Carmi & EC et CAC, petit cabinet. \\
\hline Entretien SD & Janv 2016 & $90 \mathrm{~min}$ & Abdoullah Lala & EC, membre d'un réseau. \\
\hline Entretien SD & Fév 2016 & $35 \min$ & M. Techer & Conseiller financier vousfinancer.com \\
\hline Journal de Bord & $\begin{array}{l}\text { Nov } 2014 \text { à } \\
\text { mai } 2016\end{array}$ & $\begin{array}{l}27 \\
\text { pages }\end{array}$ & $\begin{array}{l}\text { Remarques } \\
\text { personnelles }\end{array}$ & $\begin{array}{l}\text { Notes terrain avec sentiments, } \\
\text { perceptions et commentaires. }\end{array}$ \\
\hline
\end{tabular}




\section{ANALYSE DES DONNEES ET DISCUSSION DES RESULTATS}

Nous avons utilisé le logiciel N'Vivo 10, fréquemment préconisé dans les recherches en Sciences de gestion. Il facilite le codage des données, le recours à des éléments et des croisements statistiques tout en ressemblant à "l'analyse papier crayon " (Descheneaux et Bourdon, 2005), il laisse donc une place à l'interprétation du chercheur. Cette nouvelle version permet d'intégrer des pages web et les discussions sur les réseaux sociaux, fonctionnalité très utile pour les données obtenues par netnographie.

\subsection{Réputation de la plateforme et attachement du prêteur}

La réputation d'une plateforme repose sur divers aspects. Nous les présentons par ordre d'importance en fonction des fréquences d'apparition dans les données récoltées.

Le critère le plus probant est le sérieux dans le contrôle et la sélection des projets mis en ligne. Premièrement, il y a la qualité des données financières mises à disposition. Les prêteurs sont parfois méfiants des informations fournies sur la plateforme. Selon eux, le partenariat avec les EC marque une volonté de transparence de la plateforme, et démontre l'intérêt porté à leur égard : "les plateformes qui font des partenariats avec des experts financiers ont tout compris, elles veulent éviter qu'on perde de l'argent ». Les prêteurs sont rassurés par la présence de l'EC et sur les autres plateformes certains n'investissent que sur les entreprises qui doivent recourir à un commissaire aux comptes pour limiter le risque. Les plateformes affichent le nombre de projets rejetés ${ }^{17}$, afin de mettre les prêteurs en confiance.

En outre, ils accordent une importance au moins équivalente aux données de nature qualitative. Les analystes étudient «la qualité du projet ou de l'équipe dirigeante, le potentiel marché, les performances par rapport à la concurrence ». Tout est clairement exposé sur les plateformes. Lorsque les informations essentielles sont synthétisées, le prêteur y accède plus facilement, et cela l'entraîne à consulter différents projets "c'est simple et clair, on n'a pas besoin de chercher une heure ». L'investissement concernant l'ergonomie est apprécié. Ils sont sensibles à l'attractivité du site: "les couleurs utilisées rendent le site peu agréable ", " c'est la présentation de Lendopolis que je préfère : claire, agréable, très complète ». Certaines plateformes laissent aux entrepreneurs le soin de présenter eux-mêmes leur projet. Les prêteurs préfèrent les présentations sous forme vidéo. Les porteurs de projets doivent attacher une importance particulière à leur technique d'attraction de l'internaute afin de se différencier. Ces derniers désirent plus qu'une simple transmission d'information, une véritablement communication. «Ce qui est bien c'est qu'en quelques minutes on peut se faire une idée ». "Je préfère aller sur une plateforme où c'est bien expliqué, et où je peux avoir rapidement l'avis des analystes ».

Concernant les plateformes, leur objectif est d'accueillir un maximum de projets en limitant les risques. Avec le nombre de plateformes ouvertes en 2015, il paraît essentiel aux fondateurs de trouver un facteur de différenciation concurrentielle. Le partenariat de Lendopolis avec le CSOEC et le groupe les Echos en est un. «Nous nous sommes rapprochés de Lendopolis parce que cette plateforme fait l'objet d'un partenariat avec l'Ordre des experts comptables et est en contact avec mon expert-comptable. C'est également l'une des premières à se lancer sur ce secteur de l'emprunt participatif, ce qui nous semblait gage de sérieux ».

\footnotetext{
${ }^{17}$ Par exemple, sur Lendopolis au 17/06/2017, « 465 projets soumis pour 165 présentés ».
} 
Le particulier, quant à lui, prend des risques, certes, mais son taux de rétribution est très nettement supérieur à un placement classique. Il déplore le nombre trop faible de projets proposés pour pouvoir réellement faire un «choix de cour ». Sur Lendopolis, le prêteur est rassuré par l'attestation fournie par l'expert-comptable et considère ses risques minimes en rapport avec ses gains potentiels. "Lendopolis est celle où j'ai la plus forte moyenne de taux $8.4 \%$ \%. En revanche, il est difficile de répartir les risques en investissant sur une seule plateforme. Selon les experts, les taux de rémunération des prêts vont sans doute devoir diminuer, même si les entreprises sont disposées à payer pour une disponibilité immédiate des fonds: "On constate déjà une baisse des taux moyen ... On peut penser que si l'offre est supérieure à la demande, les plateformes seront incitées à faire baisser les taux ». Le particulier a tendance à renouveler ses prêts lorsqu'il considère la sélection assez rigoureuse.

\subsection{L'importance de la construction d'une confiance mutuelle}

La confiance mutuelle est le socle de toute relation durable. Dans un premier temps, le porteur de projet doit être transparent et présenter de manière attractive les informations les plus fidèles possible pour gagner la confiance des prêteurs. Sur les blogs, nous constatons le mécontentement des prêteurs qui regrettent des divergences entre les données fournies sur les plateformes et celles de la centrale des bilans. Ils peuvent également contester la note du risque estimé attribuée au projet ou regretter un certain laxisme des analystes quant à la recherche d'informations externes sur l'entreprise. Un prêteur dit sur un blog « au moins lorsque les comptes sont certifiés par l'EC, vous ne pouvez pas vous faire berner ". Son attestation d'informations financières garantit les éléments techniques communiqués, mais son rôle est également de conseiller l'entrepreneur, par exemple sur le montant de la somme à emprunter. "C'est notre métier, nous le faisons au quotidien, des business plans, du prévisionnel ». Il se base sur la connaissance qu'il a du secteur d'activité, du marché et de l'histoire de l'entreprise. S'il pense que le client ne pourra pas rembourser, il tentera de le dissuader en lui présentant les risques. Il est le plus compétent pour certifier les comptes, c'est en ce sens que sa présence dans les communautés de prêt est un atout, vecteur de confiance pour la communauté. Il apparaît complémentaire au contrôle des analystes, de par sa connaissance de l'environnement local. La situation idéale pour les membres actifs serait qu'il n'y ait aucun défaut de paiement, voire aucun retard. Les plateformes qui ont les taux de défaut les plus faibles gagnent en notoriété.

Ensuite un élément très important pour la confiance apparaît être la communication et les échanges d'opinion. "La communication avec Lendopolis est assez réactive et rassurante ". Des échanges très enrichissants entre les prêteurs, les analystes de plateformes, les gérants d'entreprises, ou encore les salariés permettent de renforcer les relations de la communauté : « Je souhaite répondre au sujet de la société GIIF, étant un ancien salarié de ce groupe et suivi la mise en place sur Lendopolis, je suis bien placé pour vous éclairer. Les articles de presse ne reflètent pas réellement le réalité "». Les prêteurs sont aussi très sensibles à la sincérité des analystes, et à leur pédagogie : "C'est assez précis et complet comme réponse je trouve. Certains devraient en prendre de la graine », un autre rétorque : "tout à fait d'accord, c'est sérieux ».

Enfin, l'aboutissement de la confiance mutuelle serait la formation d'une «intelligence collective ». La communauté confirme que seul on ne prend pas toujours les bonnes décisions, ou on ne perçoit pas toutes les subtilités mais les synergies des divers acteurs membres de la communauté l'aident à rejeter les projets douteux. "Il y a eu une intelligence collective, car le projet n'a pas été financé ». Cette dernière permettrait de détecter les fraudes, comme récemment : "Une plateforme du nom de Netfinancement.com a copié des projets de Lendix et 
d'Unilend et a appelé des particuliers pour prêter à ces projets bidons. Histoire incroyable ... cette plateforme est immatriculée à l'ORIAS en tant qu'IFP !!!! Ce qui montre que l'obtention $d u$ statut IFP est trop facile et qu'il n'existe aucune barrière à l'entrée ». La solidarité et la communication sont des éléments clés du fonctionnement des communautés de prêt. Les membres se conseillent, débâtent, se donnent des astuces et font également des analyses post financement pour informer sur le devenir de l'entreprise.

\subsection{Implication des acteurs}

Le crowdlending n'en est qu'à ses débuts et les relations se développent progressivement dans les communautés de prêt. Certains diversifient leur placement sur plusieurs plateformes. Ils regrettent la méconnaissance du crowdlending mais se montrent prêts à patienter. "Je suis complètement d'accord: si c'est pour accélérer et faire n'importe quoi pour les projets retenus, aucun intérêt ».

Les entrepreneurs financés privilégient cette alternative pour l'avenir. Ils certifient qu'ils se rapprocheront à nouveau de la plateforme lors d'un prochain projet. "J'ai encore le fichier de commandes qui se remplit, je ne vous cache pas que cette fois je ferai directement appel à Unilend ». Les entrepreneurs sont reconnaissants de l'obtention si rapide des fonds et une certaine fidélité s'instaure. Qui plus est, dans certains cas, le financement par plateforme a permis de renouer avec leur banque : «lorsque vous avez les fonds, la plateforme avertit votre banquier, il voit que les prêteurs vous ont fait confiance, et là enfin la banque m'a proposé de me prêter, à un coût plus faible ».

De plus, les récents partenariats démontrent aussi l'engouement général pour le crowdlending. L'objectif est de sécuriser le processus. La relation tripartite originelle s'élargie peu à peu. Nous avons étudié ici le cas des EC. Ils doivent s'intéresser à cette source de financement alternative pour la proposer à leurs clients. Le partenariat du CSOEC avec Lendopolis n'est pas anodin. L'EC a un rôle à jouer dans le développement du crowdlending à condition de s'y impliquer plus fortement. Un entrepreneur affirme : "Non ce n'est pas mon EC qui m'a conseillé, c'est mon gendre, il travaille à Paris dans la gestion de patrimoine, il est au courant lui! Heureusement qu'il était là en vacances, c'est grâce à lui que j'ai pu avoir mes $80000 €$ », un autre «Non ce n'est pas mon EC, c'est moi j'ai cherché tout seul, et à force de chercher j'ai découvert les plateformes ». Nous notons la reconnaissance des entrepreneurs lorsqu'ils ont été informés par un tiers de l'existence de ce type de financement «heureusement qu'il m'a dit de regarder de ce côté-là, sinon je serai encore à chercher un financement et j'aurai sûrement de grosses difficultés actuellement $»$. De nouveaux partenaires viennent enrichir la communauté de prêt, comme les assurances : "certaines plateformes proposent même une assurance antiimpayés, sous certaines conditions » ou les banques : "elles sont limitées dans leur rôle de financeur à cause de Bâle I et Bâle II et donc elles s'intéressent également au crowdlending » ce qui renforce la fiabilité du Crowdlending. Les institutionnels reconnaissent que ce phénomène digital répond à certains besoins insatisfaits jusqu'alors. Très récemment, le Medef PACA est le premier réseau professionnel régional à s'engager dans le financement participatif. Son président affirme que «la plateforme de financement participatif fait désormais partie de cette offre destinée à toutes les entreprises de nos territoires. L'intelligence collective doit primer sur toute autre considération pour les entreprises et les salariés de notre région ». Ces divers engagements (assurances, médias, investisseurs institutionnels) renforcent la communauté du crowdlending. 


\subsection{Synthèse : liens dans la communauté de prêt et réciprocité}

Nous présentons les rôles des acteurs de la communauté de prêt grâce aux 3 piliers de l'échange social : investissement (I), confiance (C) et engagement (E).

Tableau 2 : Investissement, confiance et engagement des acteurs

\begin{tabular}{|c|c|c|c|c|}
\hline & Plateforme & Porteurs de projet & Prêteurs & Expert-comptable \\
\hline I & $\begin{array}{l}\text { Sélection des projets } \\
\text { Ergonomie de la } \\
\text { plateforme et } \\
\text { praticité d'utilisation } \\
\text { Compétence des } \\
\text { analystes } \\
\text { Transparence dans } \\
\text { la communication } \\
\text { des données. }\end{array}$ & $\begin{array}{l}\text { Vérification des } \\
\text { critères d'éligibilité } \\
\text { Développement de } \\
\text { techniques de } \\
\text { promotion } \\
\text { nouvelles. } \\
\text { Echanges, réponses } \\
\text { aux questions des } \\
\text { prêteurs sur } \\
\text { plateforme ou blogs. }\end{array}$ & $\begin{array}{l}\text { Investissement } \\
\text { économies } \\
\text { Analyse et } \\
\text { sélection des } \\
\text { projets } \\
\text { Echanges avec } \\
\text { d'autres prêteurs : } \\
\text { solidarité, entraide, } \\
\text { conseils, astuces }\end{array}$ & $\begin{array}{l}\text { Attestation } \\
\text { d'information } \\
\text { financière. } \\
\text { Vérification de la } \\
\text { faisabilité du projet. } \\
\text { Relation de } \\
\text { proximité avec son } \\
\text { client. } \\
\text { Conseils }\end{array}$ \\
\hline $\mathrm{C}$ & $\begin{array}{l}\text { Réduction des } \\
\text { risques pour le } \\
\text { prêteur } \\
\text { Vérification de la } \\
\text { transparence } \\
\text { Communication et } \\
\text { réactivité }\end{array}$ & $\begin{array}{l}\text { Transmission de } \\
\text { données qualitatives } \\
\text { et financières } \\
\text { sincères. } \\
\text { Dialogue avec la } \\
\text { communauté. } \\
\text { Utilisation fidèle de } \\
\text { l'argent reçu. } \\
\text { Remboursement de } \\
\text { sa dette aux prêteurs }\end{array}$ & $\begin{array}{l}\text { Confie ses } \\
\text { économies aux } \\
\text { entreprises } \\
\text { Découverte et } \\
\text { financement } \\
\text { d'entreprises de } \\
\text { tous secteurs et de } \\
\text { toutes régions. } \\
\text { Diversifie ses } \\
\text { placements }\end{array}$ & $\begin{array}{l}\text { Individualisation du } \\
\text { conseil sur les } \\
\text { éléments à présenter } \\
\text { ou la somme } \\
\text { raisonnable à } \\
\text { emprunter. } \\
\text { Tiers de confiance, } \\
\text { Amoindri les } \\
\text { risques de } \\
\text { défaillance }\end{array}$ \\
\hline $\mathrm{E}$ & $\begin{array}{l}\text { Acceptation du } \\
\text { projet et temps de } \\
\text { collecte rapides } \\
\text { Accompagnement } \\
\text { du porteur de projet. } \\
\text { Promotion du projet. } \\
\text { Conclusion de } \\
\text { partenariats limitant } \\
\text { le risque }\end{array}$ & $\begin{array}{l}\text { Fidélité à la } \\
\text { plateforme si } \\
\text { campagne réussie. } \\
\text { Rembourse le } \\
\text { capital et les } \\
\text { intérêts. Promotion } \\
\text { pour la plateforme } \\
\text { Reconnaissance } \\
\text { envers les prêteurs }\end{array}$ & $\begin{array}{l}\text { Bouche à oreilles : } \\
\text { publicité pour la } \\
\text { plateforme et les } \\
\text { porteurs de projets. } \\
\text { Renouvellement } \\
\text { des prêts. } \\
\text { Test des produits } \\
\text { Sympathie pour } \\
\text { entrepreneur }\end{array}$ & $\begin{array}{l}\text { Réputation, } \\
\text { notoriété : mise à } \\
\text { jour de ses } \\
\text { connaissances. } \\
\text { Alternative de } \\
\text { financement } \\
\text { proposée. } \\
\text { Promotion des } \\
\text { plateformes }\end{array}$ \\
\hline
\end{tabular}

Le Crowdlending repose sur un contrat spécifique, qui au-delà de l'aspect marchand, crée du lien entre les différents partenaires. En complément du contrat économique explicite, il engendre des obligations non spécifiées. Des liens se créent entre les différents partenaires notamment par le biais des forums et des chats : entre les porteurs de projets et les membres de la plateforme d'une part, mais aussi entre les prêteurs et les membres de la plateforme, ou également entre les prêteurs et les entrepreneurs, et pour finir entre les prêteurs, qui se conseillent ou se confient leurs astuces en matière de choix. Les particuliers partagent leurs outils personnels les aidant à choisir les projets. «Voilà pour vous aider la matrice d'aide à la décision que j'ai créée et que j'utilise pour chaque projet ». Les compétences individuelles 
sont mises au profit du groupe, ce qui pourrait limiter le risque pour les prêteurs et assurer la pérennité des plateformes.

Nous présentons une synthèse des principaux éléments composant les trois obligations relatives aux échanges sociaux « donner, recevoir et rendre » (Blau, 1964).

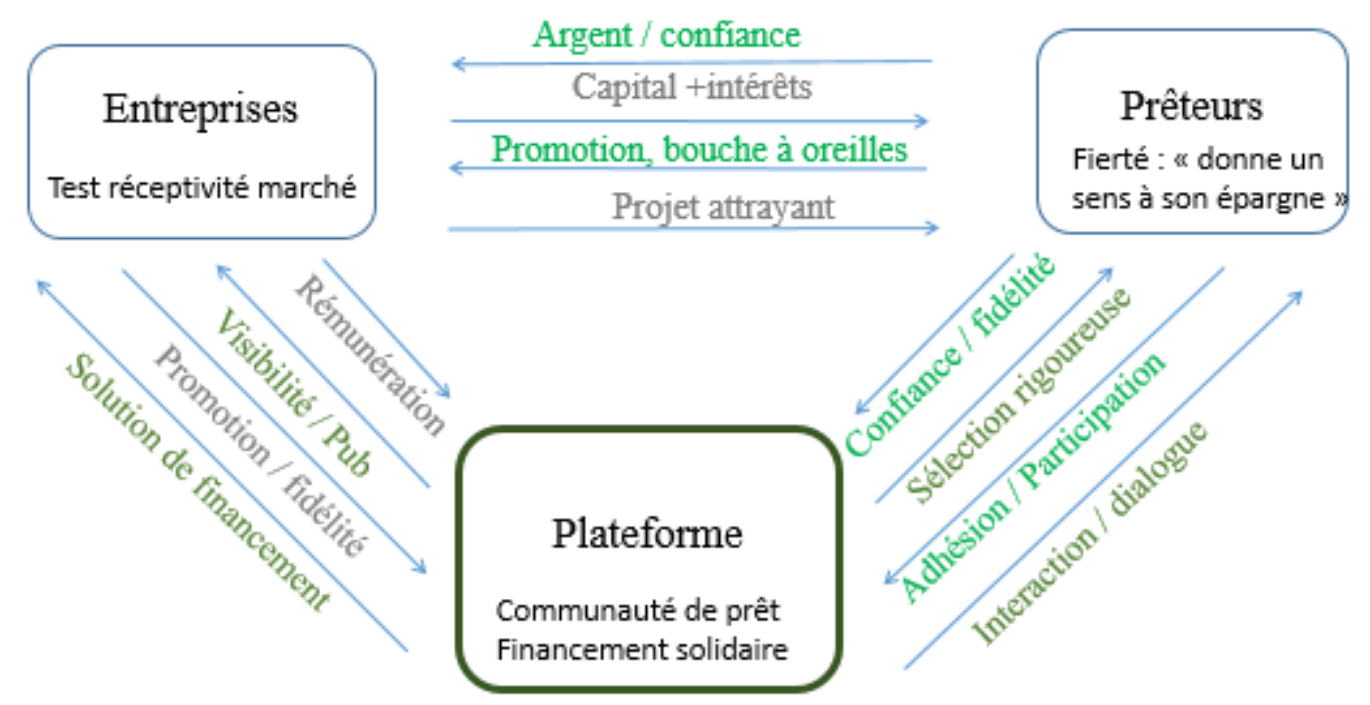

Figure 1 - Les principes de la communauté de prêt

\section{CONCLUSION}

Devant la rigidité des structures classiques, le crowdlending apporte une solution de financement alternatif. Il est considéré comme un excellent complément au crédit bancaire. Il ne sera pas le moyen de relever les entreprises en difficultés et le nombre de prêteurs actifs reste encore très limité ${ }^{18}$, mais il connaît un essor remarquable. Il en est encore à ses prémisses et ne peut pas seul répondre au besoin de financement des entreprises.

Les résultats de cette recherche exploratoire confortent que les communautés de prêts au-delà du contrat économique, apportent du lien, des interdépendances. Plus la plateforme fournira l'investissement attendu, plus les prêteurs engageront leurs économies pour participer au développement des entreprises. Le crowdlending continuera à se développer grâce à la construction d'une confiance mutuelle durable, qui passe notamment par une diminution du taux de défaut de paiement, mais également par la conclusion de divers partenariats pour élargir les parties prenantes, actrices de cette communauté de prêt. Enfin la pérennité du crowdlending et de chaque plateforme est dépendante de l'implication des acteurs à créer une intelligence collective par le biais notamment d'échanges sur la plateforme ou sur des blogs.

Sur le plan méthodologique, l'intérêt de notre recherche provient de l'hétérogénéité de l'échantillon ainsi que de l'alliance des entretiens semi-directifs et de la netnographie pour l'analyse thématique des résultats dans N'Vivo. Au total, nous avons codé 39 documents soit 198 pages et 7 heures d'entretiens vidéos. Sur le plan managérial, l'intérêt est de montrer aux

\footnotetext{
${ }^{18} \mathrm{Au} 17$ juin 2017 : 5297 sur Lendopolis, 12644 sur Unilend.
} 
dirigeants des plateformes l'importance de leur investissement tant pour la sélection vigilante des entreprises que sur leurs outils marketing, ainsi que l'importance de la confiance mutuelle pour le groupe de prêt. De nouvelles plateformes voient régulièrement le jour, il est donc important de se différencier. L'implication des membres est essentielle à la pérennité sur ce marché biface. Au niveau théorique, nous venons compléter la littérature en apportant un éclairage nouveau par l'apport de la théorie des échanges sociaux sur ce phénomène. De ces transactions qui semblent purement financières, peuvent ainsi naître des liens plus profonds de nature sociale.

Cette contribution constitue le socle de nos recherches sur le crowdlending. Une étude exploratoire est en construction pour analyser les motivations des entrepreneurs à exposer leur projet sur ces plateformes, D'autres recherches intègrent des cas d'échec pour comprendre les dynamiques qui s'instaurent lorsque les collectes n'aboutissent pas ou lorsque des retards ou défauts de paiement surviennent. En juin 2017, nous comptons 112 projets en défaut de paiement sur les 16 plateformes françaises. Une meilleure compréhension des échecs nous paraît importante pour la pérennité du modèle du crowdlending.

\section{Bibliographie}

ARTIS A. (2009). Bande dessinée et finance solidaire, destins croisés. Hermès, La Revue 2 (n ${ }^{\circ}$ 54) : 163-168

BELLEFLAMME, P., LAMBERT, T., \& SCHWIENBACHER, A. (2014). Crowdfunding: Tapping the right crowd. Journal of Business Venturing, 29(5), 585-609.

BESSIËRE V., STÉPHANY E. (2014). Le financement par crowdfunding. Quelles spécificités pour l'évaluation des entreprises ? Revue française de gestion $\mathrm{n}^{\circ} 242: 149-161$

BERNARD Y. (2004). La netnographie : une nouvelle méthode d'enquête qualitative basée sur les communautés virtuelles de consommation. Décisions Marketing n³6 : 49-62.

BLAU P., Power and exchange in social life, John Wiley \& Sons, Inc, New York, 1964.

CAUVIN E., DECOCK-GOOD C, BESCOS PL. (2006). La perception des entreprises françaises en matière de diffusion d'informations non financières: une enquête par questionnaire. Comptabilité Contrôle Audit, 12, 2 : 117-142.

CHAPELLIER P., TRIGUI T. (2007). Internet et la relations entre l'expert-comptable et le dirigeant d'entreprise. Revue française de Comptabilité, 395 : 42-48

CHOLAKOVA, M., \& CLARYSSE, B. (2015). Does the Possibility to Make Equity Investments in Crowdfunding Projects Crowd Out Reward-Based Investments ? Entrepreneurship Theory and Practice, 39(1), 145-172.

CHEKKAR R. (2005). Communication financière et analyse de discours : une réflexion méthodologique autour du cas Saint Gobain ? 26 ${ }^{\text {ème }}$ congrès de l'AFC, Lille.

COVA B. (2008). Consumer made, quand le consommateur devient producteur. Décisions marketing $\mathrm{n}^{\circ} 50: 19-27$

CROPANZANO, R., Mitchell, M. S. (2005). Social exchange theory: An interdisciplinary review. Journal of Management 31 (6): 874-900

CUSIN F. (2006) Relations marchandes et esprit d'entreprise : la construction sociale de la confiance» Revue Interventions économiques [En ligne], consulté le 12 juin 2017., http://interventionseconomiques.revues.org/766

DARDOUR A. (2015). La gestion du risque par les opérateurs de «crowdfunding » d'entreprise. Recherches en Sciences de Gestion, 2 n $^{\circ} 107$ : 45-65

DARRAS-BARQUISSAU N. (2011). Comment classer les actionnaires des entreprises cotées. Revue Française de Gouvernance des Entreprises, ${ }^{\circ} 9$ : 71-91. 
DURAN N. (2016). Le crowdlending: une opportunité de mission originale pour l'EC, communication au $37^{\text {ème }}$ congrès de l'AFC, mai, Clermont Ferrand.

DURAN N., DUBRULLE C. (2017). Les échecs en crowdlending : une étude exploratoire. Communication à la $1^{\text {ère }}$ journée d'étude sur le crowdfunding, 9 juin, Paris.

FRYDRYCH D., BOCK A. J., KINDER T., KOECK B. (2014). Exploring entrepreneurial legitimacy in reward-based crowdfunding. Venture Capital, 16(3), 247-269.

GAJDA O. et WALTON J. (2013), « Revew of crowdfunding for development initiatives », Evidences on demand.

GALOIS I. et LACROUX A. (2010) La fidélité dans l'intérim est-elle possible ? Une approche par les théories de l'échange social et du contrat psychologique, Revue Sciences de Gestion $\mathrm{n}^{\circ} 74 \mathrm{p}$ 89-106

GOLIC Z. (2014). Advantages of crowdfunding as an alternative source of financial of small and medium-sized entrerprises. Proceedings of the faculty of economics in East Sarayevo: $39-48$

HAAS P., BLOHM I., LEIMEISTER JM (2014), An empirical taxonomy of crowdfunding intermediaries, $35^{\text {th }}$ International conference on information systems, Auckland.

HOWE J. (2006). The rise of crowdsourcing. Wired.

KLEEMANN FG., GUNTER V., KERSTIN R.(2008). Un(der)paid Innovators:The commercial Utilization of Consumer Work through Crowdsourcing. Science, Technology and Innovation Studies, vol $4 \mathrm{n}^{\circ}$ 1: 5-26.

KOZINETS R. V. (2002), The field behind the screen : using netnography for marketing research in online communities. Journal of Marketing Research, 39 : 61-72

KUTI M. et MADARASZ G. (2014). Crowdfunding, Public Finance Quaterly, vol 59 ; p 355366

LAMBERT, T., \& SCHWIENBACHER, A. (2010). An empirical analysis of crowdfunding. Retrieved 11 20, 2013, from SSRN : http://ssrn.com/abstract=1578175.

LANGEVIN P. et MENDOZA C. (2014), Impliquer les managers à atteindre leurs objectifs : participation, feedback et confiance, revue CCA, tome 20 vol 3, p 43-71

LEROY J. (2008). Gestion de la relation avec une communauté virtuelle dans une stratégie de co-création : les leçons du cas Fon.com, Décisions Marketing, n52 : 41-50.

MAUSS M., «Essai sur le don. Forme et raison de l'échange dans les sociétés archaïques », dans Lévi-Strauss C., Sociologie et anthropologie, PUF, Paris, 1950, p. XVI-XVII.

MOLLICK, E. (2014). The dynamics of crowdfunding: An exploratory study. Journal of business Venturing, 29(1), 1-16.

ONNEE S., RENAULT S. (2013). Le financement participatif : atouts, risques et conditions de succès. Gestion, n ${ }^{\circ}$ spécial créativité, vol 38, n³ : 54-65.

ONNEE S., RENAULT S. (2014). Crowdfunding : vers une compréhension du rôle joué par la foule ? Management et Avenir : 117 - 133.

POETZ MK., SCHREIER M. (2012). The value of crowdsourcing : Can Users Really compete with professionals in generating new product Ideas ? Journal of Product Innovation Management, Vol 29, $\mathrm{n}^{\circ} 2: 245-256$.

RENAULT S. (2014). Crowdsourcing compétitif : ressorts en enjeux. Recherches en Sciences de Gestion, $\mathrm{n}^{\circ} 101$ : 59-80.

ROCHET JC et TIROL J. (2006), Two sides markets : a progress report, Journal of economics, 37(3), p 645-667

SOULARD O. (2015). La crédibilité des avis en ligne : une revue de littérature et un modèle intégrateur, Revue Management et avenir, n82, Dec., p 129-153

VALANCIENE L. et JEGELEVICIUTE S. (2013). Valuation of crowdfunding : benefits and drawbacks, Economics and Management, ${ }^{\circ} 18: 39-48$ 